\title{
Variations
}

Variations

Revue internationale de théorie critique

$21 \mid 2018$

L'industrie de la culture : version originale

\section{Histoire et subjectivité rebelle. Au sujet de Negt et Kluge}

\section{Fredric Jameson}

Traducteur : Claire Debard

\section{(2)enEdition \\ Journals}

\section{Édition électronique}

URL : http://journals.openedition.org/variations/896

DOI : 10.4000/variations.896

ISSN : 1968-3960

\section{Éditeur}

Les amis de Variations

\section{Référence électronique}

Fredric Jameson, «Histoire et subjectivité rebelle. Au sujet de Negt et Kluge », Variations [En ligne],

21 | 2018, mis en ligne le 05 avril 2018, consulté le 19 avril 2019. URL : http://journals.openedition.org/ variations/896 ; DOl : 10.4000/variations.896

Ce document a été généré automatiquement le 19 avril 2019.

Les ami•e•s de Variations 


\title{
Histoire et subjectivité rebelle. Au sujet de Negt et Kluge
}

\author{
Fredric Jameson
}

Traduction : Claire Debard

\section{NOTE DE L'ÉDITEUR}

Nous remercions Fredric Jameson de son aimable autorisation pour l'édition française de son article, initialement paru dans la revue October, vol. 46, Alexander Kluge : Theoretical Writings, Stories, and an Interview, 1988, MIT, pp. 151-177.

Un grand merci à José Chatroussat pour sa relecture pointue.

Les sous titres de la version française ont été ajoutés par la rédaction de Variations.

1 Neuf ans séparent la publication d'Espace public et expérience (Öffentlichkeit und Erfahrung) de celle d'Histoire et subjectivité rebelle (Geschichte und Eigensinn), les deux ouvrages écrits en collaboration par Oskar Negt et Alexander Kluge ${ }^{1}$. Ce qui frappe d'abord le lecteur «matérialiste» (au sens où il lit des livres physiques, plutôt qu'en termes d'«idées »), c'est à quel point ils témoignent de la révolution typographique qui s'est produite entre leurs deux parutions - avec le postmodernisme, la fin des années 1960 et la défaite de la gauche. Le premier livre souffre manifestement des contraintes formelles du discours classique. Au travers de ses six chapitres conventionnels qui cherchent à fonder une théorie de l'espace public " prolétarien ", leurs auteurs se retrouvent obligés, à l'encontre de leur volonté, de fournir à la place les rudiments d'une théorie de l'espace public bourgeois. Ici, tout le contenu a déjà commencé à migrer vers les notes de bas de page et les annexes : trois «digressions » et une vingtaine de « commentaires » séparés forment en l'occurrence le tiers d'un volume de cinq cents pages où quelques illustrations commencent déjà à émerger. 


\section{L'héritage de Walter Benjamin}

2 Ailleurs, dans les diverses zones théoriques du "Premier monde", de nouvelles idéologies de l'hétérogène et de la différence ont commencé à inspirer des notions « rhizomatiques » de la forme : les «plateaux » deleuziens sont disposés côte à côte dans des chapitres distincts et sans lien apparent, tandis que les deux austères colonnes de Glas vous mettent au défi de comprendre à quel moment passer de l'une à l'autre. Mais de manière encore plus catégorique, les discontinuités dans les récits et les films de Kluge interdisent tout retour à l'essai ou au traité traditionnel, la route se trouvant obstruée suite à une chute de gravats («Vous pouvez imaginer le problème du réalisme antagoniste à la façon dont on analyse l'état d'un lieu où une explosion s'est produite. Celle-ci a dispersé des objets sur une vaste étendue. La force de l'explosion, autrement dit celle qui les a réellement déplacés, n'est plus présente...»[p.348]). Au niveau généalogique, les «constellations dialectiques» de Benjamin ou d'autres montages comme les idéogrammes de Pound - semblent présenter un air de famille, même si l'« amoncellement des images » suggère encore avec force chez ces deux prédécesseurs une bonne façon de tout mettre ensemble. Pourtant, l'esthétique propre à Kluge (et celle de Geschichte und Eigensinn, qui a quelque chose d'un film théorique) est résolument postbenjaminienne plutôt que post-brechtienne. En dépit de la longue et étroite collaboration entre Kluge et Adorno, leur dernier ouvrage a pour ancêtre l'énorme et fragmentaire Passagen-Werk de Benjamin, ou du moins ce qu'on imagine que ce dernier projet aurait pu devenir. Voici par exemple ce que dit Kluge de l'un de ses films :

[Il] ne produit pas des énoncés, mais des proportions ; c'est un objet sur lequel on peut argumenter. Notre point de départ réside dans l'observation qu'il n'y a pas de forme immédiate d'expérience sensible, ou tout du moins aucune forme organisée susceptible d'englober les divers domaines individuels du travail et des milieux de production. Seul un espace public factice, comme on en trouve notamment dans les médias, peut présenter un tel ordre et une telle unité... La question se pose ainsi : comment doit-on procéder avec une réalité ainsi désordonnée, avec un mélange d'expériences? Comment apprend-t-on au milieu des erreurs? Comment pouvonsnous faire face à des impressions objectives et subjectives déformées...? Vous devez prendre la réalité comme une matière brute... A notre avis, les spectateurs peuvent se servir de ce film pour tester leurs propres conceptions de ce qui est public et de ce qui est réaliste ${ }^{2}$.

Cependant, la segmentation dans les récits de Kluge ne relève pas seulement de la perspective et du découpage cinématographique (avec une séquence de quinze minutes d'expériences juxtaposée à un paragraphe condensant huit années); elle provoque aussi des sauts qualitatifs dans des dimensions incommensurables. Cette expérience particulière de lecture se prolonge dans Histoire et subjectivité rebelle où des notes sur le «mode de production » de Marx (qu'on voit somnoler la plus grande partie de la journée sur son canapé tandis que les gens entrent et sortent, écrire des commentaires méchants dans les marges et éparpiller ses feuilles maculées de taches de tabac), des considérations sur le Blitzkrieg ou la Chanson de Roland, des illustrations tirées de la théorie de l'évolution et de l'histoire des automates, des anecdotes sur Kant, des citations de lettres à Fliess, des études du travail domestique, l'histoire des prix, la politique des romantiques allemands, des lectures au pied levé de contes de fées, tous ces éléments se succèdent dans un ordre imprévisible et rivalisent avec une extraordinaire collection de centaines d'images tirées de manuscrits médiévaux, de films, de journaux ouvriers, de publicités, de graphiques, de 
modèles scientifiques, de photos d'actualités, d'images de mobilier ancien, d'illustrations de science-fiction, d'exercices de calligraphie et de la reconstitution de voies romaines ou de batailles de la Renaissance. Les divers chapitres, sections, paragraphes, notes et digressions (qui suivent différents modes de numérotation) sont reclassés typographiquement en alternant les caractères, les encadrés et les encadrements; ou alors, de façon encore plus spectaculaire, les pages noires aux caractères blancs sont intercalées entre des expériences plus « normales » (donnant parfois l'impression, comme l'alternance de la couleur et du noir et blanc dans la série Heimat réalisée par l'ancien chef opérateur de Kluge, Edgar Reitz, que c'est le changement même qui importe, et non une correspondance univoque régulière entre le contenu et le mode de représentation: Proust en a dit autant sur l'alternance de l'imparfait et du passé simple chez Flaubert).

L'autorité est ainsi déplacée et transformée; la lecture reste un exercice, un entraînement, une socialisation et une pédagogie, mais dans ce travail, il ne reste que peu de chose de son côté terrorisant ou disciplinée, pas même l'impératif dialectique de l'ancien montage où, comme chez Godard, on reste mis au défi de trouver ou d'imaginer le bon point de vue. Ici, les écarts et les sauts suggèrent un processus associatif qui diffère du nôtre, ou du moins la confiance suggère l'existence d'une alternative qu'il pourrait être intéressant d'essayer d'approcher, sinon d'apprendre. De fait, l'importance accordée à l'apprentissage est à tel point omniprésente que nous nous trouvons ainsi bien disposés à envisager la possibilité utopique d'établir des relations entre thèmes et objets exposés - ce qui ne correspond pas au style personnel ou au domaine méthodologique de Negt et Kluge, mais reste à inventer.

\section{Expérience, production, capital}

5 Pourtant, comme Negt et Kluge ne se lassent pas de nous le rappeler, l'expérience de la production doit être distinguée de ses instruments ou de ses produits avec lesquels elle est incommensurable : l'économie politique, la logique du capital (capitalogic), traite de cette expérience, mais il est plus difficile, et plus tortueux, de chercher comme ici à formuler une « économie politique de la force de travail » (p. 139). Cela signifie aussi qu'il sera structurellement contrariant de chercher à expliquer ce livre, comme nous devons le faire ici, au moyen des différentes «théories " qu'il convoque en passant, en revenant patiemment à un "système" qui se voulait d'abord un moyen d'action, voire une habitude, au sens fort et positif du terme. Penser (y compris la «théorie », synonyme de marxisme dans tout le livre) est ici réduit de façon thérapeutique à une composante de l'action, qui est elle-même considérée comme une forme de production, ainsi que nous le verrons bientôt.

6 Il convient, arrivé là, d'enregistrer une réserve similaire à propos du langage, et plus particulièrement des mots que nous employons pour désigner des concepts envers lesquels Negt et Kluge ont adopté des positions assez peu canoniques. De fait, l'histoire de la pensée moderne peut être racontée sous la forme d'une exploration des conséquences d'un scepticisme linguistique radical, où la sophistication philologique de Nietzsche et l'attaque de la langue ordinaire par Sartre dans La Nausée culminent paradoxalement avec l'importance privilégiée accordée au langage dans le structuralisme et le poststructuralisme, qui entérine le diagnostic et confirme sa fonction (sous des formes allant de la syntaxe occidentale aux réseaux kantiens ou aux épistèmes discursifs) de nouvel équivalent de l'idéologie elle-même et comme source de toute erreur. Cependant, 
cette formulation est tout à fait trompeuse dans la mesure où elle implique la possibilité de la vérité (c'est-à-dire la possibilité de l'atteindre en dehors du langage lui-même). Le problème de la production de concepts philosophiques dans ces conditions rejoint progressivement le problème du statut d'un nouveau langage ou discours "théorique ", dont tout ce qu'on peut dire est qu'il doit être radicalement provisoire et par conséquent s'abolir lui-même au cours du processus. Aujourd'hui, les discussions critiques aussi influentes les unes que les autres de l'essentialisme et de l'anti-essentialisme ou de l'antifondationnalisme seraient mieux appréhendées comme des mises en accusation des principaux codes linguistiques plutôt que comme des « croyances » dont personne ne sait avec certitude si elles existent ou non. Cependant, que la mission de la philosophie aujourd'hui soit accomplie ou ne puisse pas l'être, elle semble aller de pair avec le problème d'assurer la mortalité de son langage.

7 Une impulsion analogue, bien qu'inverse, est à l'œuvre dans la tradition allemande de la spéculation philosophique que Jean Paulhan a appelée la " preuve par l'étymologie ${ }^{3}$ ", à savoir l'étude des racines et des radicaux de l'allemand contemporain à la recherche de traces d'un mode plus ancien et plus primordial de la relation à l'être lui-même. On a justifié cette procédure au motif qu'elle établirait une relation avec la langue tribale plus directe, sans mélange et sans médiation, que celle qui survit dans les langues romanes ou en anglais par exemple ; et elle permet à la philosophie allemande de faire valoir sa parité avec la Grèce où Socrate (ou Platon) argumentait souvent d'une manière semblable, transformant l'« étymologie populaire » en un boulevard pour la raison philosophique (on trouve une analogie semblable, bien que plus éloignée, en Chine où le caractère écrit offre des preuves semblables de significations plus anciennes et " plus vraies »). L'abus de tels arguments par Heidegger rend embarrassant leur reprise dans la pensée de gauche de Negt et Kluge (penser - begreifen - vu comme apparenté à greifen, saisir ou empoigner dans le processus de production [pp. 20-22]) jusqu'au moment où l'on comprend qu'ils ne font pas référence à la "nature" ou à l'«être", mais à ce que Marx appelait le naturwüchsig, autrement dit à la structure sensiblement différente de formations sociales antérieures plus simples. D'autre part, cette «méthode » n'a rien à voir avec la solennité religieuse des rituels stylistiques de Heidegger :

Dans la partie réalisée par Kluge de Deutschland im Herbst, on voyait déjà Gabi Teichert en train de sonder l'histoire allemande à l'aide d'une bêche. Ces scènes ont été reprises dans Die Patriotin, où le fait de creuser à la recherche du passé et de l'histoire de l'Allemagne est devenue la métaphore centrale. L'expression figurée, « creuser à la recherche des trésors du passé ", est prise au pied de la lettre par Kluge qui la restitue visuellement sous la forme d'une image concrète d'une fouille physique dans la terre gelée. Il en résulte une sorte d'image-jeu de mots dans la tradition de Buñuel ou Karl Valentin, qui a pour effet de tenir le spectateur à distance afin de l'amener à observer les excentricités de Gabi Teichert avec plus de scepticisme critique que d'empathie. Il en va de même lorsqu'elle traduit le savoir accumulé dans d'épais livres d'histoire en une perception sensorielle et «travaille » dans cet état d'esprit sur de vieux in-folio, ce qui est aussi illustré « au pied de la lettre »: elle dissèque les livres d'histoire à l'aide de scies, de perceuses et de marteaux et dissout leurs pages dans du jus d'orange afin de les étouffer. Elle s'« ouvre » ensuite de la même manière " une voie dans l'histoire ", elle " assimile l'histoire en elle ", etc. - autant d'images oniriques irréalistes fondées sur des figures de linguistique. Lorsqu'elle participe en tant qu'archéologue à temps partiel à des fouilles illégales de l'ancien rempart de la ville, elle espère « saisir » [be-greifen] le passé sous la forme d'outils 
préhistoriques, c'est-à-dire être en mesure à la fois de le "prendre " et de le « comprendre $»^{4}$.

Deux autres observations s'imposent au sujet de ces « efforts » pour rétablir la pureté du langage philosophique par une nouvelle intervention ou par l'archéologie linguistique. Indépendamment de ce que la crise du discours philosophique doit à l'incertitude métaphysique ou ontologique du langage lui-même, elle peut aussi être lue en termes socio-économiques comme le résultat local d'une marchandisation accrue qui fait entrer les termes philosophiques abstraits (considérés un peu aujourd'hui comme la propriété privée ou comme des marques de leurs créateurs) dans le champ de force de la réification où leur transformation de plus en plus rapide en objets et images culturels détruit tout aussi rapidement leur légitimité philosophique (et celle de la philosophie elle-même). C'est aussi ce que cela veut dire quand des concepts philosophiques sont considérés comme « démodés » ou « dépassés » (une réaction qui aurait paru très étrange au sein de la philosophie traditionnelle); c'est également ce que Paul DeMan semble avoir pensé dans sa réflexion sur la "thématisation » qui est le lot réservé aux thèmes et concepts philosophiques à l'époque moderne.

D'autres solutions restent néanmoins possibles dans la prolifération actuelle des codes et discours théoriques éphémères ou provisoires, à savoir des solutions qui ne proposent pas (d'une façon démodée ou dépassée) l'invention de codes et de discours plus vrais, ni le retour à des codes et discours plus purs. C'est le cas, par exemple, de la notion de «transcodage» en tant qu'alternative contemporaine à la critique philosophique traditionnelle. Elle implique en l'occurrence que les différents " termes principaux » ou " codes principaux» gouvernent des zones distinctes du réel, souvent contiguës et chevauchent les zones du réel, auxquelles ils donnent leur nom, de sorte que l'alternance systématique de ces termes ou la comparaison de leurs capacités signifiantes ne donne pas lieu à l'émergence d'une nouvelle synthèse linguistique ou terminologique, mais à une sorte de relevé des matières premières qui constituent le réel (la "substance" linguistique de Hjemslev). Le processus est analogue au problème de la traduction dans le domaine des langues naturelles qui toutes présentent, au moins à un niveau minimal, des éléments comparatifs distincts du sens qu'une phrase traduite est censée partager avec la phrase originale. Ce qui est philosophique dans la traduction n'est donc pas la tentative de reproduire un énoncé en langue étrangère comme étant le même, mais plutôt l'expérience plus profonde qu'elle offre de percevoir les différences radicales entre les langues naturelles.

\section{Espace public et expérience}

11 Le transcodage s'impose d'emblée avec le premier livre de Negt et Kluge, Öffentlichkeit und Erfahrung (1972) dont le titre ne peut être traduit qu'imparfaitement en anglais par Public Sphere and Experience $e^{5}$. Ce qui motive le choix de l'équivalent anglais est assez clair dans la mesure où le substantif publicity a été annexé depuis longtemps par un secteur spécialisé de ce plus vaste domaine public rendu par le mot allemand öffentlichkeit, tandis que la notion de «sphère » ou « espace » - qui peut être transposée dans d'autres dimensions de la vie sociale telles que la culture - pose des questions théoriques intéressantes par ellesmêmes (ce qui n'est pas le cas du terme allemand). En même temps, on peut dire que ce thème en lui-même " appartient » en quelque sorte à Jürgen Habermas dont le premier ouvrage Strukturwandel der Öffentlichkeit (The Structural Transformation of the Public Sphere 
,1962) retrace l'histoire de l'émergence des institutions des premiers médias bourgeois, y compris leur théorisation philosophique et juridique, depuis une perspective qu'on retrouve inchangée dans toute son œuvre, à savoir que les valeurs de la révolution bourgeoise restent universelles, de sorte qu'elles ne sauraient être analysées sous forme d'idéologie fonctionnelle d'une classe sociale particulière. Les limites et les échecs tangibles de ces valeurs ne sont par conséquent, pour Habermas, ni internes, ni structurelles, mais résultent plutôt du blocage historique du processus révolutionnaire bourgeois, demeuré incomplet et non-réalisé. Negt et Kluge ne partagent pas cette perspective puisque pour eux, la tendance à monopoliser l'espace public à l'époque moderne est intimement liée à la fonction de classe du concept bourgeois de public et à la nature des institutions qui en sont issues. C'est pourquoi ils proposent et défendent un type radicalement différent d'ouverture et de communication collectives qu'ils appellent «l'espace public prolétarien ${ }^{6}$ ».

Le transcodage va cependant au-delà de la simple traduction. Pour en apprécier la portée première à sa juste valeur, il faut revenir au second terme dans le titre de Negt et Kluge, Erfahrung, ou "expérience", afin de prendre pleinement la mesure de ce que sa signification a de plus profondément implicite, à savoir que le concept d'" espace public » gouverne une partie de la vie sociale bien plus vaste que chez Habermas. En effet, ce dernier a tendance, dans sa première œuvre, à réduire l'espace public aux institutions spécialisées des médias naissants (journaux, opinion publique, débat « représentatif » ou parlementaire, etc.), tandis que ses développements philosophiques ultérieurs (actes de parole, agir communicationnel) montrent bien à quel point il se méfie des concepts phénoménologiques tels que l'expérience - ou tout autre de la sorte de l'autre côté du Rhin. Negt et Kluge peuvent donc être placés aux côtés des anti-structuralistes qui défendent la notion d'expérience et vont de E. P. Thompson et Raymond Williams à Sartre, mais avec des différences et modifications inattendues comme cela deviendra plus clair lorsque nous examinerons la poursuite de cette idée et en estimerons la valeur dans leur second ouvrage.

Le développement de la notion d'espace public par Negt et Kluge a néanmoins ceci de significatif que, sans cesser d'inclure les référents institutionnels de l'histoire d'Habermas (sous leurs formes contemporaines comme la télévision), ils cherchent à élargir la notion de manière à garantir son lien constitutif avec la possibilité même de l'expérience sociale ou personnelle en général. L'« espace public » est désormais considéré dans sa structure sociale comme ce qui permet l'expérience, mais aussi, d'un autre côté, ce qui la restreint et y met un frein. Cette structure détermine aussi la pathologie moderne fondamentale qui coupe en deux l'« expérience » elle-même, avec ses deux moitiés inégales assignées, d'une part, à des expressions publiques stéréotypées et, d'autre part, à cette zone personnelle et particulière qui semble protéger du public et du politique alors qu'en même temps, elle est elle-même un fait social né du public et du politique. Dès le départ par conséquent, Öffentlichkeit devient comparable à un " concept convenu » qui rivalise avec de multiples autres concepts, de la « cure par la parole » de Freud à la notion et au langage même de la "démocratie ", sous sa forme politique autant que sociale. \{0> ("Workplace democracy," for example, now constitutes a central and ineradicable space of the "proletarian public sphere," and the political stake in transcoding can now be measured by way of a comparison between the relative weight of the political rhetoric of "democracy" and that of the new discursive space of öffentlichkeit.) 子ło $_{3}$ (La « démocratie sur le lieu de travail » par exemple occupe désormais une place centrale et indéfectible 
dans l'« espace public prolétarien » et l'enjeu politique du transcodage peut désormais être mesuré au moyen d'une comparaison entre le poids relatif du discours politique de la « démocratie » et celui du nouvel espace discursif de l'öffentlichkeit).

${ }_{00\}}$ L'originalité de Negt et Kluge réside dans la manière d'accroître, pour ne pas dire de compléter, la force critique et analytique de la pratique universellement connue comme $l^{\prime}$ «analyse du discours» (notamment dans les descriptions que fait Foucault des restrictions et exclusions à l'œuvre dans toute une série de formations dites discursives) par la tentative utopique de créer un espace discursif d'un type nouveau. Mais cela repose le problème philosophique de la création d'un nouveau langage ou d'une nouvelle terminologie pour l'associer à la question de l'espace public; car il existe des raisons sociales et historiques qui justifient l'absence d'un nouveau langage philosophique plus approprié, c'est-à-dire d'un nouveau langage public. Les formes et expériences auxquelles ce langage correspond n'existent pas encore. L'absence même d'un espace public prolétarien complique par ailleurs la tentative de lui donner un nom, autrement que dans les lacunes de notre discours actuel. Il en va de même de la conception du «travail » et de la « production » que Negt et Kluge tentent d'élaborer dans Histoire et subjctivité rebelle ( Geschichte und Eigensinn) et qu'ils décrivent comme une " économie politique de la force de travail [Arbeitsvermögen] » (pp. 136-143). Or, même dans le marxisme, ces mots désignent une zone restreinte ou spécialisée de l'activité humaine : le travail, la main d'œuvre ou la production n'existent que dans la mesure où ils peuvent être « réalisés » en tant que tels (comme dans «la reproduction de la force de travail de l'ouvrier »). Dès les premières pages du Capital en effet, la séparation inaugurale de la valeur d'usage et de la valeur d'échange (et l'utilisation consécutive de ce terme pour désigner uniquement la seconde) montre que Marx écrit une économie politique du travail, une logique du capital, et non l'anatomie de ses dessous démiurgiques, l'anthropologie de la force de production humaine tentée ici. Öffentlichkeit und Erfahrung a été en ce sens un échec: nous avons entrepris, nous disent Negt et Kluge, le projet d'un espace public prolétarien et nous nous trouvons réduits à rédiger une critique des limites de l'espace public bourgeois. Le terme Öffentlichkeit rejoint cependant dans leur travail ultérieur le concept culminant d'un « espace public commercial industriel» (Produktionsoffentlichkeit) historiquement nouveau, identifié à l'histoire elle-même.

C'est pourquoi le second ouvrage transcode en partie le premier. Mais il pose un problème discursif plus fondamental à la doxa actuelle, non seulement du fait de son engagement permanent en faveur de la catégorie de l'expérience (comprenant la dimension anthropologique de la description de toute une série de "capacités" physiques, psychologiques et culturelles), mais surtout du fait de la prédominance totalement hors de propos qu'il confère à la catégorie de production en soi (que les auteurs conçoivent très différemment des usages à la mode, métaphoriques et souvent culturels qui en sont faits au cours de la période althussérienne et post-althussérienne). Un concept de production absolutisé à ce point et étendu à toutes les activités humaines est-il encore "productiviste » au sens négatif du terme? Le jugement sera plus juste s'il est basé sur la réussite de l'expérience langagière elle-même et sur la capacité d'un langage productiviste à formuler une grande variété de matières normalement régies par d'autres langages ou codes, en particulier dans le domaine de la psychanalyse, du désir, de l'imagination, de l'intime, de l'inconscient, mais aussi ces réalités d'un ordre très différent que nous appelons histoire, ou faits historiques (et ici spécifiquement l'histoire allemande). Deleuze et Guattari (auxquels nous faisons parfois référence ici) nous ont 
familiarisés avec ce type d'expériences dans le premier de ces domaines. Le second pourrait sembler quelque peu contradictoire avec ce que nous avons appelé la dimension anthropologique de ce travail, étant donné que les anthropologies philosophiques (en particulier lorsqu'elles postulent l'« agressivité » ou une « volonté de puissance » comme une composante de la nature humaine, à l'instar de la sociobiologie) comportent généralement un engagement implicite envers les positivismes et placent par conséquent la violence et les catastrophes de l'histoire hors de leur portée.

Le problème de l'histoire est assurément présent dès le titre du second ouvrage de Negt et Kluge, mais pas encore le concept de production (ni même celui de force de travail) qui, curieusement et par substitution, est "représenté » par le mot intraduisible Eigensinn. Miriam Hansen l'a rendu en anglais par "entêtement" (obstinacy), mais aussi « autonomie » (autonomy), tandis qu'Andrew Bowie en fait un «sens délibérée » (willful meaning) ${ }^{7}$. J'y ajoute ma suggestion : « volonté propre » (self-will), qui redonne sa place à la composante "propre » ou " propriété originelle» et équilibre l'accent (parfaitement justifié) mis sur l'arbitraire et l'entêtement, avec la connotation parallèle d'une logique immanente, une pulsion ou impulsion à rester fidèle à soi-même et suivre sa propre ligne de force indépendante, sa propre trajectoire spécifique qui est alors, comme dans la lecture que fait Bowie du terme, son sens même. Ces explications ont pour but de faire disparaître les sous-entendus auxquels le mot self («propre ») avait donné lieu à tort dans ma version. Non qu'il ne soit pas question d'«identité » dans ce texte, mais cette identité, collective ou individuelle, ne sera atteinte que plus tard: c'est le propre, le soi pour ainsi dire du Gesamtarbeiter de Marx, ou du travailleur collectif, et non d'une notion actuelle ou antérieure de la construction du sujet (notamment la structure freudienne dans laquelle Negt et Kluge suggèrent que les fonctions psychiques agissent de la même manière que la représentation parlementaire bourgeoise [p. 382]). Ce n'est donc pas un « soi » primitif doté d'Eigensinn, mais plutôt un vaste ensemble de compétences, pulsions et capacités acquises et développées au cours de l'histoire et dont chacune a ses propres exigences « entêtées » et son « sens » spécifique. Ces forces peuvent être résiduelles ou émergentes ; elles ne peuvent souvent pas être utilisées à pleine capacité et cette inactivité génère des pathologies spécifiques, de même que leur répression, leur aliénation ou leur détournement. Ce qui génère en revanche une pathologie sociale, c'est leur multiplicité, c'est-à-dire la possibilité permanente de contradictions ou d'une coordination néfaste entre elles. C'est ce qui explique par exemple le cas (si souvent mis en scène par Kluge dans ses fables) où une " capacité ", qui est à elle seule une puissante force de la nature, soumise aux hasards de l'histoire ou associée à d'autres forces de même intensité, peut avoir des effets quasi-mortels, voire mortels.

\section{Après les avant-gardes}

Ce premier appel à une logique plus profonde et plus significative - sinon des instincts humains, du moins des pulsions et forces humaines d'origine sociale et historique - porte implicitement un rejet des politiques de gauche d'avant-garde. Ce rejet est explicite dans les deux ouvrages de Negt et Kluge. L'Eigensinn (subjectivité rebelle), ou la force de travail, la capacité de travail, s'y rapproche du "bon sens " de Gramsci, il est inhérent à la collectivité et n'a guère besoin de stimulations intellectuelles ou politiques d'avant-garde. Si ce «bon sens » semble néanmoins souvent incomplet, c'est l'effet d'un conservatisme naturel plus profond de l'organisation humaine, apparenté aux exigences d'abri, de protection et de subsistance. Les deux livres consacrent de fait un grand nombre de pages 
à l'analyse systématique des crises historiques du mouvement ouvrier dans cette perspective ; elle est évidemment essentielle au projet de Negt et Kluge qui, s'il pouvait encore faire appel à certaines conceptions de la révolution culturelle en 1972, reflète une situation de découragement et de pessimisme de la gauche en 1981. Les deux auteurs n'en revendiquent pas moins un type d'espoir à plus long terme, d'ordre géologique ou évolutionniste, un espoir qui retient de la révolution culturelle ses impulsions pédagogiques ou son incitation à l'auto-formation et l'auto-reconstruction.

Geschichte und Eigensinn est articulé en trois vastes sous-sections: "L'Organisation historique de la capacité de travail »; «L'Allemagne en tant qu'espace public commercial et industriel [Produktionsöffentlichkeit]» et «La Puissance relationnelle (ou «de la relationalité ») [Gewalt des Zusammenhangs] ». La description qui suit est sujette à caution, bien qu'indispensable : la première partie met en place les différents éléments de ce que j'ai appelé l'« anthropologie » de Negt et Kluge, à savoir leur « économie politique de la force de travail» - qui s'intéresse au processus de travail, mais aussi à des questions évolutionnistes et à la coexistence de nombreux rythmes et cycles temporels (individuels, historiques et biologiques). La deuxième partie tente de faire face aux "particularités » de l'histoire allemande avec ces nouvelles catégories de production: ses problèmes de forme viennent de l'écart conceptuel entre le langage des faits historiques et une conception de la production dont l'échelle et l'objectif diffèrent clairement de l'historiographie avec laquelle ils sont souvent perçus comme sans commune mesure. Quant à la dernière partie - qui comprend aussi une étude approfondie de la guerre comme une sorte de production, et une longue discussion de l'expérience existentielle et de questions psychanalytiques - la meilleure manière de l'appréhender est d'y voir une tentative de créer une nouvelle valeur éthique et politique active qui soit également un concept d'analyse opératoire, à savoir celui de la relation ou de la relationalité en soi. Des positions théoriques émergent dans chacune de ces longues parties et je vais essayer d'en restituer certaines, même si les philosophes pourraient objecter qu'elles ne sont pas étayées par des arguments et si leur présentation ne prend plus la forme d'un traité de philosophie ou d'un essai discursif. Il est peut-être plus juste de décrire le livre comme un genre de film conceptuel (si l'on entend par « film » l'un de ceux réalisés par Kluge).

Le concept médiateur essentiel de la partie d'introduction « anthropologique » - qui doit être suffisamment abstrait pour fonctionner avec des matières très différentes, mais aussi porter en lui la force d'un évènement (un traumatisme, un changement, une cicatrice, une transformation, une modification irrévocable qui ouvre de nouvelles possibilités) - est la notion marxienne toujours classique de Trennung ou " séparation, division »; soit la "séparation » historique qu'on trouve surtout chez Marx entre producteur et moyens de production (comme entre objet produit et production en elle-même résultant de ma propre activité). Pour Marx, c'est bien évidemment l'élément structurel central de la catastrophe historique à l'origine du capitalisme, à savoir la soi-disant "accumulation primitive ». Il existe donc déjà chez Marx un lien entre forme de production et fait historique. Negt et Kluge vont alors projeter le fait - l'accumulation primitive - et son concept structurel - la Trennung, la division et séparation - dans un fait historique et philosophique plus général qui englobe toutes les catastrophes historiques, les plus décisives étant celles des débuts, la destruction des sociétés agricoles et communautaires traditionnelles. Le concept de séparation est alors applicable à d'autres questions abordées dans la littérature marxiste traditionnelle : la division du travail, la séparation entre travail manuel et travail intellectuel, la fragmentation du psychisme en «facultés » 
distinctes, et enfin la notion de réification elle-même (qui, chez Lukacs, est essentiellement une question de "taylorisation » de la vie sociale). Pour Negt et Kluge, l'accent semble mis avant tout sur la séparation des différentes forces de travail ou capacités, avec des conséquences qui seront explicitées plus tard.

On objectera que ce type de contexte tend implicitement ou explicitement à valoriser le phénomène d'« unification » dont il dépend nécessairement. C'est possible - et leur vision de la vie communautaire dans les campagnes pourrait témoigner d'une nostalgie historique - mais Negt et Kluge rejettent explicitement toute conception de la dialectique visant à rétablir une unité primitive ("Quelle réalité aurait la réappropriation de quelque chose de perdu?»[pp. 42-44]). C'est pourquoi, loin de perpétuer le regret de la réunification, le fait et le concept de Trennung auront les effets très différents de générer de la relationalité en tant que telle, la formation permanente de nouveaux liens et de nouvelles relations. Ce point aussi trouve une analogie formelle dans Marx, avec l'émergence de la valeur et de la relation sociale historiquement neuve qu'il appelle coopération, issue de la catastrophe historique du travail salarié industriel (p. 192 et voir ci -dessous).

\section{Travail mort, travail vivant}

21 L'autre concept qui émerge de cette notion élargie et généralisée d'«accumulation primitive » concerne ce qui est accumulé : dans l'« économie politique de la force de travail », ce sera le «travail mort », le travail accumulé, le travail humain du passé - un mystérieux capital d'activité humaine de production considérablement associée par Marx aux machines et à l'industrialisation, qui font bondir d'un seul coup l'accumulation de temps de travail caractéristique de l'histoire humaine plus ancienne. Tel qu'il est ici plus largement développé par Negt et Kluge, le « travail mort » désigne à la fois la tradition en général, le capital culturel et l'habitus (pour reprendre la terminologie de Bourdieu), il comprend la reproduction de caractéristiques acquises, de structures de caractère archaïque et les niveaux historiques du psychisme. Le concept de travail mort est cependant inquiétant ${ }^{8}$ pour les deux auteurs et peut justifier les violences de l'histoire et ses désastres irréversibles d'apparence cyclique (évitant ainsi la tentation idéologique et anthropologique de postuler l'existence de forces négatives au sein de la «nature humaine » telles que l'agressivité et la volonté de puissance). Avec cette connotation négative de la notion de travail accumulé, ils se rapprochent de l'idée sartrienne du "pratico-inerte» (développée dans la Critique de la raison dialectique) selon laquelle la pratique humaine engagée avec succès dans la transformation de l'objet monde se retourne "par magie » contre les êtres humains avec une force autonome propre sous une forme destructrice aveugle et la désormais incompréhensible et antihumaine « contre-finalité » d'une histoire qui échappe à tout contrôle humain. Toute comparaison entre ces deux projections philosophiques voisines doit tenir compte de la prépondérance différente, entre la catégorie centrale que fait Sartre de la pratique en tant qu'activité humaine réalisée de quelque type qu'elle soit, et la notion développée par Negt et Kluge de la force ou capacité de travail qui souligne la potentialité, la formation souterraine et l'exercice de multiples capacités. La vision de Sartre de la contre-finalité est incomparablement plus spectaculaire et plus frappante que celle de Negt et Kluge, mais elle est aussi relativement monolithique et subordonne un grand nombre de troubles historiques au seul concept désigné. Chez Negt et Kluge cependant, le travail mort peut présenter de multiples résultats historiques: dans l'histoire allemande c'est le 
traumatisme originel de la Guerre des Paysans, mais aussi le démantèlement initial de la production communautaire à l'aube de la féodalité et la grande « occasion manquée » de la guerre de libération nationale contre Napoléon en 1811.

Mais évaluer ces faits historiques implique de mettre d'abord en place une lecture des «modes de production » marxiens. L'attention accordée par Negt et Kluge aux différents modes de production est typiquement guidée par leur intérêt pour les capacités et les formes de force de travail spécifiques que chacun de ces modes de production développe. Leur premier schéma (pp.165-210) isole trois moments de production différents: l'agriculture, l'artisanat et le travail industriel. Les spécifier comme des types de travail distincts (et comme des dominantes historiques différentes ou des moments de développement social différents) revient à imaginer comment des capacités requises et développées sous une certaine forme sont susceptibles de subir une sorte de sédimentation sous la forme suivante, laissant des traces et des cicatrices sur les couches de subjectivité comme sur le corps, sur l'expérience et dans l'histoire elle-même. Tels les muscles d'un nageur, mobilisés pendant la guerre pour creuser des tranchées et utilisés après-guerre pour lui permettre de gagner sa vie en tant qu'acrobate de cirque, les capacités développées au cours de l'histoire demeurent, inutilisées, mal utilisées ou réadaptées, elles interfèrent entre elles à l'occasion ou, de façon symptomatique, marquent et déforment les gestes courants d'un nouveau genre de quotidien.

Pour Negt et Kluge, le travail de la terre est clairement la forme «naturelle » de la vie sociale humaine, ou plutôt (car ce type d'affirmation est étranger à leur travail), c'est la forme la plus ancienne, la fondation autant que le point de départ de la formation sociale européenne, et plus spécifiquement allemande :

L'ampleur des potentialités du travail aux champs peut uniquement être mesurée par le biais de ses transformations modernes. Tous les producteurs actuels descendent d'ancêtres appartenant à la classe paysanne, de sorte qu'il existe en quelque sorte un " paysan en moi » pour chacun. Cette composante de la capacité de travail contemporaine - à certains égards sa base - se révèle (distinctement de ses représentations historiques) pleine de ressources [wendig], fonctionnant de manière non compartimentée, fournissant des visions et des indices de vie collective plus concrets que les modes de production ultérieurs. En effet, la composante subtile de l'activité intellectuelle à proprement parler suit la logique d'un mode de production paysan ou jardinier. La capacité de travail qui vise un processus d'émancipation ou une prise de conscience économique doit nécessairement déployer la vision d'une propriété originale issue de l'histoire agricole. L'idée des qualités «naturelles» d'un produit et le développement de mesures humaines du temps et de la temporalité sont eux aussi dérivés de cette même source. En revanche, le travail agricole actuel dans notre pays est un sous-ensemble du processus industriel. (p. 174)

Ce serait exploiter à mauvais escient ou mal comprendre cette observation que de l'utiliser pour développer une vision idéologique conservatrice ou nostalgique du passé. Elle pose plutôt une question empirique à propos de l'imagination utopique effectivement existante et, par-là même, de la possibilité de développer une vision politique du changement et de l'action. Ce qui est défendu ici et dans tout le livre, c'est qu'Eigentum désigne quelque chose de plus fondamental et nécessaire que la propriété (property, le sens littéral du mot), au sens juridique de formes de propriété privée qui prennent naissance au cours de l'histoire et peuvent être abolies. Eigentum, - à présent au sens plus étymologique de ownness, ce qui m'appartient ou ce qui nous appartient en propre, ce qui informe Eigensinn - le terme n'est pas une question de possession, mais de lieu et d'espace, 
et de notre relation à ce que Marx appelait « le corps de la terre ». La conséquence pour Negt et Kluge est que la vision d'une utopie purement urbaine est impossible. ${ }^{9}$ L'imagination utopique devra toujours s'accommoder d'une manière ou d'une autre des exigences de cette couche la plus ancienne de la conscience ou capacité de travail. L'urgence de la question politique - car aucun mouvement ou pratique politique authentique ne peut se développer sans une vision de l'avenir et d'un changement radical - n'est pas la seule qu'il convient ici de souligner, mais bien aussi qu'à l'époque post-moderne, caractérisée par l'atrophie de l'imagination utopique en général et de la capacité d'avoir un projet pour l'avenir en particulier, l'analyse du fonctionnement de l'imagination utopique figure en bonne place à l'ordre du jour.

Par contraste, la dévalorisation de l'artisanat par Negt et Kluge doit être interprétée en opposition à une situation où les intellectuels sont en règle générale fortement attirés par ce mode d'activité qui semble offrir une idéalisation de leurs activités professionnelles, de l'écriture et de la production culturelle en général ${ }^{10}$ (on trouve une discussion plus complète des auteurs quant aux capacités de production plus générales à l'origine du travail des intellectuels au chapitre « À propos de certaines omissions frappantes dans le fonctionnement de l'intelligence ", pp. 415-488). On peut alors considérer que l'artisanat transmet des valeurs d'art et de métier dont le côté plus sombre est moins souvent mis en avant - pas uniquement l'extension du travail à des produits non naturels, pas même la « libération » tendancielle du temps et de l'espace, de la terre et des saisons, mais surtout un principe de concurrence historiquement nouveau qui découle du travail des petits producteurs, dont chacun met un point d'honneur à différencier son activité de celle des autres. Ce principe de concurrence détermine une extension générale de la production... Cette extension tendancielle est profondément enracinée dans l'artisanat, jusqu'à l'autodestruction. Les corporations essaient donc par nécessité de modifier cette impulsion en limitant les produits et en restreignant le choix des métiers et professions possibles. (p. 175)

27 Les valeurs même de l'artisanat mènent donc dialectiquement à la concurrence et à un mouvement sans fin vers la surproduction qui annonce l'économie de marché, ses structures et ses rythmes. L'artisan doit travailler à deux choses en même temps : (1) son produit et (2) à convaincre le client que son produit est indispensable et particulièrement utile. C'est une conviction que l'ouvrier agricole n'a pas spécialement besoin de susciter. Tandis que si le travail artisanal n'affiche pas la preuve d'un travail méticuleux et ne témoigne pas délibérément de sa présence, l'activité cesse d'être considérée comme un métier et d'être une profession viable. L'honneur professionnel doit être présent dans l'objet, tel un paiement pour lui - un premier paiement en reconnaissance de son style et de sa spécificité, suivi d'un second paiement, celui en argent. (p. 176).

C'est pourquoi les jalousies entre professionnels et les fiertés, la concurrence et la dynamique sans fin de l'accumulation de marchandises sont déjà implicites dans ce mode de production, de même que les vices sont inhérents à ses vertus. Le mode industriel et la nature du travail industriel requièrent moins d'attention car ils ont déjà fait l'objet d'un examen approfondi par Marx lui-même. Quant à Sartre, dans un esprit très différent de celui de Negt et Kluge, sa perspective essentiellement urbaine l'a souvent entraîné à célébrer la conscience antinaturelle et post-naturelle générée par le travail sur des machines - une insistance qui constitue clairement un élément essentiel de tout "ouvriérisme " ou de toute idéologie "ouvriériste» conséquente. Les formes spécifiques de l'aliénation du travail en usine, et notamment ses divisions spécifiques ou 
Trennungen, sont plus nettement restituées dans les théories contemporaines, en particulier l'analyse fondamentale que fait Harry Braverman du taylorisme et de ses effets. Cependant, ce type d'analyse, dont la pertinence pour la culture et le travail intellectuel n'a pas échappé aux théories contemporaines, n'est pas particulièrement mis en valeur par Negt et Kluge, malgré leur description frappante d'un sous-segment de production dans une aciérie (pp. 207). La coopération est la seule capacité nouvelle sur le plan historique que développe le travail en usine, et c'est l'émergence de cette nouvelle forme de capacité de travail qui permet à Negt et Kluge de rédiger la dernière partie de leur ouvrage sous l'angle de la relationalité qui en découle.

Les modes de production sont en général discutés et débattus sur un plan plus historique, en fonction de différents systèmes : la féodalité, le capitalisme, le mode de production asiatique, etc. Pour comprendre ce qui rattache la discussion précédente à ce type de catégorie historique et comment la guerre représente parfois une caricature sinistre du travail coopératif à l'échelle nationale, nous devons maintenant passer par le « chas de l'aiguille » de l'histoire allemande (pour reprendre l'une des expressions favorites de Negt et Kluge). Elle a le même point de départ que les histoires évoquées plus haut, à savoir la primauté de l'expérience paysanne et des capacités spécifiques développées à partir du travail de la terre. En même temps, l'expérience allemande est déterminée par la situation géographique de l'Europe centrale qui présente toutes les caractéristiques d'une collectivité terrestre enclavée. Or, si le mot expérience a un sens, ce n'est pas uniquement par rapport aux problèmes, dilemmes ou crises auxquels nous sommes confrontés, mais aussi par ce que nous apprennent les solutions récurrentes et par ce qui est transmis sous forme d'habitude et de pédagogie. Mais où trouver les traces de cette expérience et le codage de ces processus d'apprentissage collectif?

\section{Désirs utopiques}

Dans les contes de fées, qui ne sont pas simplement les dépositaires des désirs utopiques des paysans (« ceux qui ne croient pas aux contes n'ont jamais connu la détresse » [p. 619, n. 48]) mais conservent les expériences collectives les plus caractéristiques de dangers ou de menaces, ainsi que les solutions séculaires imaginées pour y parer. En Allemagne, les contes sont un témoignage collectif équivalent, même s'ils sont sensiblement différents, aux mythes et légendes épiques de marins du monde méditerranéen classique auxquels il est très instructif de les comparer. Car les activités, les compétences, les forces et les capacités célébrées, immortalisées et transmises par les récits grecs - ces «vertus » bien connues que sont la sagacité et la ruse, l'ingéniosité et l'habileté, dont Ulysse est l'exemple-type - sont aussi les attributs professionnels d'un univers de commerce et de négoce, de ruse marchande et de diplomatie impériale, attributs incarnés par les bateaux, complétés par l'ultime recours à la mer, la fuite à la voile ou le retour de nuit, les avirons assourdis. Pour les paysans cependant, ces récits posent problème et sont inutilisables : contrairement aux grands navires, « la maison, la cour de ferme et le champ ne peuvent échapper aux dangers qui les menacent » (p. 752). Mais du point de vue d'un conte paysan où «l'ampleur de la production (ou son appauvrissement) constitue le moment déterminant", les récits d'exploration et d'aventures maritimes peuvent être vus différemment et leurs perspectives inversées ${ }^{11}$. Les protagonistes du mythe des Argonautes, par exemple, sont, du point de vue grec, Jason et son équipage ; et ils sont dans une position centrale qui relègue l'expérience paysanne - la population terrienne de Colchide - dans la position de l'Autre : elle est en l'occurrence le prix et l'objet de 
l'exploitation, ce n'est pas à elle que s'adresse le récit. Ce retournement radical de la perspective paysanne est particulièrement manifeste dans l'inhumanité et la monstruosité dont fait preuve le personnage de Médée qui, du point de vue indigène (comparer avec les rôles joués par La Malinche ou Pocahontas dans le Nouveau monde), adopte les attributs du patriote et de la guérilla, de Judith et des batailles des guerres de libération nationale. Dans cette perspective, les Argonautes ne sont pas de simples aventuriers. Ils ont pour fonction de favoriser les échanges et le développement du marché, de sonner le glas de l'ancien système agricole et communautaire: «divers épisodes mis bout à bout équivalent à ce qu'est pour la production, la séparation de la force de travail de la terre et de la communauté, à savoir l'accumulation primitive. Dans la perspective de la répartition des biens ou de la relation d'échange, c'est un marché analogue d'apprentissage forcé qui émerge, d'introjection par la violence » (p. 747).

$31 \mathrm{Au}$ sein du monde paysan, et plus particulièrement du monde des contes de fées allemands, cette violence du commerce, cette "ouverture" forcée et cette menace extérieure est restituée sous des formes narratives très différentes et exige de développer des compétences essentiellement défensives très différentes. "Le Loup et les sept chevreaux » notamment, accorde une grande importance à la capacité de discernement ou de jugement :

On ne sait plus avec certitude qui est l'ennemi. Il devient excessivement difficile de juger si la patte couverte de farine du loup ou la voix haut perchée appartiennent ou non à la mère des chevreaux, ni si la mère (fin ou moyens) ne pourrait pas elle aussi avoir le poil couvert de farine ou une voix haut perchée, et ainsi de suite. L'esprit est dirigé, non vers l'histoire en soi, mais vers une question bien plus essentielle : comment être sûr de reconnaître l'ennemi, où sont les limites entre intérieur et extérieur, entre sécurité et insécurité ? Tous les mythes allemands, témoignages de l'expérience historique, ont pour contenu la question du comment décider, et font le récit de cette incertitude centrale : Comment en savoir plus sur l'extérieur depuis l'intérieur ?... Cela montre aussi à quel point il était difficile, sur la base de la relation de l'Allemagne à l'histoire, de vérifier ce qui était laissé à l'intérieur avec Hitler (pp. 754-755).

L'originalité de ces analyses de Negt et Kluge, qui constituent un prolongement inédit et imprévisible des réflexions de Benjamin sur le récit collectif dans Le Conteur, réside dans une herméneutique qui, tout en tenant compte de la fonction du conte consistant à refléter doublement la situation collective - à la fois négativement et positivement (englobant l'espoir utopique et inscrivant la catastrophe historique) - s'élance vers une troisième voie interprétative, relisant le texte comme une pédagogie collective, comme le vecteur de transmission, non seulement de l'expérience, mais aussi d'une formation professionnelle collective. Le rappel instinctif de Brecht, comme toutes les fois où la fonction pédagogique de la littérature est invoquée à l'époque contemporaine, ne doit pas nous détourner des différences fondamentales d'accent. En effet, le contenu brechtien diffère fortement de l'insistance de Negt et Kluge sur l'apprentissage des compétences et des capacités. La lecture que fait Brecht des textes du passé insiste le plus souvent sur la mauvaise pédagogie, dans l'esprit d'une critique idéologique négative (voir les magnifiques sonnets sur Hamlet et sur Kleist). Cela ne veut pas dire que pour Negt et Kluge une leçon, même bien apprise, ne peut pas avoir des conséquences mitigées :

Le prototype du conte qui essaie de retravailler l'expérience historique [aucun rêve idéal de bonheur n'est favorisé par les faits sociaux eux-mêmes] pour le rendre propice à l'accomplissement des souhaits est «La Belle au bois dormant »: une méchante sorcière - ou en tout cas une sorcière rendue méchante par la 
déception et qui n'est donc pas simplement méchante, une camarade en un sens - a plongé la princesse et le château avec tous ses habitants et serviteurs dans un sommeil enchanté. Ils sont désormais entourés d'une haie impénétrable. Mais une bonne fée a aussi prononcé un vœu tout au début, etc., etc. L'essentiel est d'avoir la patience d'attendre les mille ans que dure le changement. Par conséquent, lorsqu'il s'avère que le prince ressemble à Bismarck, Hindenburg, Hitler ou Adenauer, ce n'est pas par bêtise que l'on fait l'erreur de croire au baiser qui réveille, ni par manque d'expérience (certains princes sont visiblement très âgés, d'autres viennent de lointaines régions périphériques de l'empire et n'ont que très peu de sang bleu), mais plutôt par besoin urgent de conférer une expression objective (bien qu'improbable) au travail immémorial en cours d'accomplissement des souhaits. Cela doit être appliqué, et applicable, d'une manière ou d'une autre. Même si ceux qui prétendent être princes sont la plupart du temps des imposteurs. (p. 619 $\left.n^{\circ} 48\right)$ culturelle, retient l'importance du monde paysan que ses crises endémiques et les dangers confrontent à l'habitus spécifique de la capacité de travail développée par la vie paysanne, ce qui ne revient évidemment pas tout à fait au même que la mission d'en venir à bout. La même histoire est ensuite racontée de nouveau avec des références aux différents modes de production historiques - et en particulier sous l'angle de la crise féodale - sur un ton beaucoup plus concret qui donne leur apparence formelle aux faits et aux catastrophes historiques. Negt et Kluge proposent une analyse provocante de la féodalité (pp. 559-565), suggérant que c'est la nature essentiellement impure ou mélangée des féodalités qui s'y sont implantées qui donne aux autres situations et histoires nationales leur dynamisme productif - leur capacité à ne pas simplement « évoluer » vers le capitalisme, mais à générer des mouvements politiques actifs de toutes sortes. Les différents types de féodalités qu'on trouve en Italie, en France et en Angleterre ne sont de fait jamais indigènes ou autochtones, ils sont le résultat de diverses interventions extérieures - en Italie les empereurs germaniques, en France les Francs, en Angleterre les Normands. Ces importations marquent le nouveau système socio-économique, à tel point que ses propres contradictions internes spécifiques ne peuvent s'enraciner et qu'il devient sujet à des modifications et changements historiques radicaux. Negt et Kluge suivent cependant Marx dans la partie des Grundrisse souvent rebaptisée "Modes de production précapitalistes » et voient la féodalité comme une conséquence dialectique, mais en aucun cas organique, de la structure communautaire des tribus germaniques. C'est pourquoi elle revêt en Allemagne une forme très différente des autres histoires en Occident, ce qui explique que «le principe capitaliste n'a trouvé aucune introjection originale [originäre Verinnerlichung] dans notre pays » (p. 893) :

La règle de base est la suivante : là où une formation sociale naît, elle continue de porter en elle toutes ses contradictions et la radicalité (die Gründlichkeit) de sa naissance. Elle n'évolue donc pas systématiquement vers sa forme la plus accomplie, car ces mêmes contradictions et radicalités mettent à mal par définition son principe absolu. En ce sens, ce n'est pas un prototype original, mais plutôt la structure féodale que Guillaume le Conquérant et ses barons ont amenée avec eux en Angleterre, qui représente la réalisation la plus parfaite d'une constitution féodale classique (pp. 562-563).

Transposée dans le débat actuel des «modes de production", l'analogie pourrait être l'histoire même du mode capitaliste dont la forme «indigène » en Angleterre n'a été notoirement accompagnée d'aucun développement politique «pur» de la bourgeoisie triomphante et d'un État des classes moyennes (comme en France). 
Quel que soit le jugement rendu sur cette nouvelle théorie de la "transition", il aura deux séries de conséquences différentes pour Negt et Kluge. L'une concerne ce qu'on appelle souvent le caractère national : « Dans la configuration allemande de la féodalité/ forme de capitalisme, le principe d'abstraction [pour Negt et Kluge comme pour la tradition marxiste en général et pour Adorno en particulier, une description plus générale de la «logique du capital » essentiellement abstraite] apparaît comme principe d'incertitude dans l'application du pouvoir; de même, son principe de production spécifique peut être décrit comme un idéal d'exhaustivité et de profondeur [Gründlichkeit] (p. 564). Nous reviendrons brièvement à cet habitus qui associe l'arbitraire à une éthique du travail compulsif.

résultat, historique, des contradictions de la féodalité germanique est un fait : la catastrophe qu'a représenté la Guerre des Paysans, le traumatisme originel de l'histoire allemande et l'une des « résolutions » de la lutte des classes à laquelle Marx pensait sans doute lorsqu'il évoque dans le Manifeste, comme alternative à la «transformation révolutionnaire de la société toute entière ", la possibilité de «la ruine commune des classes en lutte ${ }^{12} \%$. En effet, c'est très exactement à cette ruine commune qu'a abouti la Guerre des Paysans :

La Guerre des Paysans se termine par la victoire politique d'une coalition de citoyens des villes et de seigneurs féodaux, mais d'un point de vue économique, les trois classes qui ont participé à la révolte - paysans, seigneurs, villes - subissent toutes les trois une défaite. Par la suite, aucune des trois ne parviendra jamais à établir une domination politique indépendante. Les questions politiques et économiques les éloigneront les unes des autres (p. 556).

Negt et Kluge insistent sur les conséquences culturelles de la catastrophe et il est logique, au vu de l'importance qu'ils accordent à la terre et au travail des paysans, qu'ils soient amenés à les dissocier comme un ultime symptôme de la stigmatisation et de la répression dont a été victime la culture paysanne en Allemagne au cours des siècles suivants, donnant naissance à une culture artificielle basée sur ce que Bourdieu appelle la "distinction». Même les classes moyennes émergentes souhaitent aujourd'hui être "raffinées", avoir des manières, transcender le corps et acquérir la "culture » et le " goût » dans leurs nouvelles significations idéologiques. Un autre conte, celui des « Trois frères » est édifiant à cet égard, montrant l'absurdité de la compétition pour de nouvelles compétences arbitraires alors que tous finissent par vivre de nouveau ensemble dans la vieille maison. Il met en scène la distance qui sépare la vie collective, la demeure simple sur un lopin de terre, de l'activité «simple». Car la simplicité n'est pas un but facile à atteindre. Avoir son propre terrain et une vie collective correspond plutôt à une structure psychologique complexe et reste le point culminant et le prix auxquels toute force de travail s'efforce de parvenir. À l'échelle de l'homme ou de l'individu, ces valeurs sont encore plus éloignées et difficiles à obtenir, c'est pourquoi il faut travailler plus dur encore et se donner plus de mal. Tel est précisément le mode de développement de l'introversion de la capacité de travail en Allemagne (p. 632).

C'est alors le moment où la tendance au rejet de la culture paysanne, le refus radical de la «capacité de travail» paysanne et le refoulement du «paysan en moi " génèrent dialectiquement la forme allemande du principe d'abstraction mentionné plus haut (qui est pour Negt et Kluge un thanatos virtuel ou une pulsion de mort au sein du capitalisme comme de l'histoire allemande). L'accent ne porte pas sur les caractéristiques d'une identité nationale ou d'un caractère national (qui prend alors une sinistre et unique place dans le pluralisme des cultures humaines et des identités collectives), mais plutôt sur 
l'échec de l'identité nationale et ce que les auteurs appellent la "perte nationale " (Nationalverlust, p. 538), considérée non en termes de psychologie collective, mais comme une sous-catégorie d'une perte générale de réalité (voir ci-dessous). C'est ici qu'émerge une forme spécifique de l'«inflexibilité» allemande aux multiples incarnations historiques et culturelles, et aux nombreuses expressions linguistiques: de l'éthique du travail à la minutie la plus profonde (sich Mühe geben, Gründlichkeit, voir ci-dessus) à la terrible droiture du Michael Kohlhaas de Kleist (et de Luther et Kant) et, au-delà, à l'inflexibilité (Unerbittlichkeit) du ralliement de dernière minute à l'État dans les dernières semaines de la Deuxième Guerre mondiale, ou l'implacable stratégie de la Fraction armée rouge de Baader et Meinhof au milieu des années 1970 : fiat justitia pereat mundus! (« que la justice soit, le monde dût-il s'effondrer ») :

Même si la société civile devait se dissoudre avec le consentement de tous ses membres (par exemple si la population d'une île décidait de se séparer et de se disperser dans d'autres régions du monde), il faudrait d'abord exécuter le dernier meurtrier encore en prison afin que chacun reçoive ce qu'il mérite et que la population ne soit pas coupable d'un crime capital pour n'avoir pas tenu à ce qu'il soit puni... "Mieux vaut qu'un homme meure plutôt que tout un peuple vienne à être corrompu. »Car si la justice disparaît, la vie des hommes sur terre n'a plus aucun intérêt. ${ }^{13}$

\section{Eigensinn, subjectivité rebelle, entêtement} centre de Geschichte und Eigensinn sous la forme du plus court et du plus atroce des contes de Grimm, «L'Enfant obstiné » (Das eigensinnige Kind) dont l'entêtement, exprimé d'abord sous forme de désobéissance, puis d'une maladie à caractère psychosomatique, persiste après la mort (une main s'obstine à sortir de la tombe) et doit être châtié à titre posthume par la mère avant de trouver le repos. Dans la partie consacrée à l'histoire allemande, le mystérieux Eigensinn du titre de Negt et Kluge (interprété philosophiquement dans les diverses traductions plus haut) prend désormais un sens littéral ironique, d'une part en posant la question du rapport de l'histoire et de la volonté inflexible en Allemagne-même, mais aussi en soulevant le problème du rapport au passé et à la mort dans le pays, et notamment la question qui a fait couler beaucoup d'encre du «travail de deuil» ( Trauerarbeit selon l'expression de Freud) qui serait nécessaire pour exorciser ce passé (plutôt que de le refouler). Cependant, en ce qui concerne ce conte en particulier, l'équivalent grec prend la forme d'un reproche (pp. 765-769) : l'entêtement d'Antigone est aussi une forme héroïque de résistance politique dont la résonance sociale et collective est totalement absente de l'histoire allemande. S'il est certain que son Eigensinn reste mortel, c'est une issue qui, Hegel l'a montré, a reçu la consécration sous forme de tragédie, c'est-à-dire d'une contradiction et d'un blocage inéluctable du développement historique. On n'y trouve pas trace de la honte qui colle étrangement au conte de Grimm où l'entêtement de l'enfant semble pathologique, mais où même la mère (chez Negt et Kluge une pourvoyeuse de nourriture et de refuge, associée à la communauté primitive) prend un caractère singulièrement ambivalent et repoussant lorsqu'elle brise le bras de l'enfant mort avec son bâton.

Ce qu'il convient maintenant de montrer en conclusion, c'est comment la troisième partie du livre, consacrée à la relation, la relationalité en tant que telle (Zusammenhang), permet de diagnostiquer ces symptômes et fournit une prescription pour les transcender. Avant tout, il est particulièrement urgent d'évoquer ces domaines traditionnellement dissociés 
que sont le public et le privé ; le politique et le psychologique ; la socio-économique et son langage de production, et la psychanalyse et son langage de désir et d'imagination. L'acte de les mettre en relation sera, ainsi qu'en témoignent de nombreux courants de la pensée contemporaine, un acte ponctuel et discontinu, un échange provisoire d'énergies, une étincelle qui traverse les frontières qui séparent. Les tentatives plus anciennes de synthèse formelle freudo-marxienne qui intègrent tant bien que mal les découvertes de Freud au discours de Marx pour former un système entièrement nouveau ne sont pas répétées ici. À la base du caractère provisoire des nouvelles approches "relationnelles ", on trouve sans aucun doute le sentiment que les dimensions objectivement dissociées dans notre ordre social ne peuvent pas, finalement, être réassemblées et réunies par un effort de pure pensée.

\section{Corriger Habermas}

Là aussi, en un sens, Negt et Kluge prolongent et corrigent Habermas dont la " synthèse " de Marx et Piaget ${ }^{14}$ visait à substituer une évolution cognitive à la violence discontinue de la révolution sociale. Chez Habermas, les questions du désir, de l'inconscient et de la sexualité n'occupent plus la première place, mais la psychologie de Piaget permet encore dans son œuvre de jeter pour ainsi dire un pont entre la situation historique et sociale "objective» et le "facteur subjectif», à savoir les dispositions individuelles et l'équipement mental nécessaires au changement social et à l'inauguration d'une nouvelle phase du développement social. Chez Negt et Kluge, la référence à Piaget n'en est qu'une parmi d'autres au sein d'une myriade d'exemples analogiques ou illustratifs, notamment Freud, la théorie de l'évolution, l'anatomie, l'archéologie culturelle et tout le reste. Le grand thème cognitif cher à Habermas - transformé ici en thème pédagogique et formateur - est cependant maintenu au centre et offre une réponse utopique à la critique classique des "lumières" bourgeoises en tant que telles par l'école de Francfort (une critique toujours gênante pour Habermas puisqu'il a tenu à promouvoir les possibilités utopiques inhérentes précisément à ces lumières bourgeoises et à la conception bourgeoise de la raison).

En ce qui concerne le désir et l'imagination, leur position au sein de la théorie contemporaine semble venir du sentiment très répandu que la narration, l'image, l'imagination, le symptôme incarné ne sont plus désormais des épiphénomènes subjectifs, mais des composants objectifs de notre monde social, empreints de toute la dignité ontologique des matières sociales présentées jusqu'à présent comme "objectives " par l'économie, la politique et l'historiographie. Plus significatif encore, on considère aujourd'hui de plus en plus que ces phénomènes subjectifs ou psychologiques possèdent des fonctions épistémologiques, ou même pratiques. L'imagination n'est plus considérée comme une réponse personnelle de compensation à des situations publiques, mais comme un mode de lecture de ces situations pour les penser et en dresser le plan, pour intervenir, même si c'est sous une forme très différente des réflexions abstraites de la philosophie ou de la politique traditionnelles. Les deux volumes de Deleuze et Guattari, Capitalisme et schizophrénie (qui viennent si souvent à l'esprit à la lecture de cette autre collaboration de deux auteurs qui nous occupe) attestent de la richesse de ces explorations, de même que les Männerphantasien de Theweleit (cités tous les deux dans cet article). Avec la notion traditionnelle de Raison cependant, l'une des victimes de cette revalorisation de l'imagination (qui reflète sans doute des changements historiques à la 
fois de la structure de la société et de l'appareil médiatique du capitalisme tardif) est la conception traditionnelle de l'idéologie et de l'analyse idéologique (surtout celle de la fausse conscience, ou le modèle infrastructure-superstructure auquel il est cependant peu fait appel ici). De nombreuses nouvelles « idéologies du désir » se sont engouffrées dans la brèche ouverte par cette disparition. Il est également certain que, fidèle aux positions politiques anti-avant-garde de leurs auteurs, le travail utopique de Negt et Kluge rejette implicitement le négativisme de certaines formes traditionnelles de «critique idéologique "; on se rend compte, notamment en observant leur collaboration théorique, parallèlement aux récits et aux films de Kluge, que c'est la primauté du «facteur subjectif» qui est ici partout affirmée - mais en tant que fait historique. Tout comme Marx attribuait au capital lui-même un Heisshunger (appétit vorace) pour la réalisation de la valeur, Negt et Kluge identifient un appétit comparable et un désir pour le "privé », l'« intime » et le « subjectif » dans la société moderne :

Les relations sont à trouver dans tous les espaces publics ou les espaces structurés sous forme d'enclaves privées. Mais de tous les rapports sociaux, ce sont les relations libidinales que renferment les contacts personnels au sens strict du terme qui atteignent les niveaux d'intensité les plus déroutants... La désintégration de l'espace public traditionnel programmée dans le système de crise actuel ne débouche donc pas uniquement sur le renforcement des forces consacrées à la construction d'espaces publics alternatifs ou prolétariens. Elle se heurte simultanément à l'autre tendance à l'accumulation personnelle du travail relationnel [Beziehungsarbeit], à une sorte d'appétit vorace pour le travail relationnel, à la recherche personnelle du bonheur (p. 877).

43 Cette lecture compensatoire de la subjectivation de la vie moderne est cependant complexifiée chez Negt et Kluge par leur insistance (propre à la période post-années1960) sur l'importance de l'« intimité » exprimée par des phrases telles que: «L'intimité est la pierre de touche pratique pour la substance de l'espace public» (p. 944). C'est cette insistance qui fait le lien entre les contenus de Geschichte und Eigensinn (Histoire et subjectivité rebelle) et l'espace public prolétarien dont on affirme à présent que celui-ci peut seulement être testé (politiquement autant que théoriquement) par sa capacité à transformer toutes les matières premières du privé ou de l'intime (ou, pour reprendre notre terminologie plus étroite, à le transcoder).

Le premier ouvrage, qui traitait nécessairement en détail de la marchandisation de l'imagination dans les médias contemporains (y compris dans le fascisme) adoptait une position populiste quelque peu simplifiée sur la transformation du subjectif en nouvel espace public prolétarien. S'appuyant sur la notion de "code restreint» de Basil Bernstein pour le langage de la classe ouvrière (que Bernstein voit comme essentiellement dépendant de la situation) et guidé par une critique voisine du " principe d'abstraction" dans les médias bourgeois, Öffentlichkeit und Erfahrung insiste sur les exigences physiques et sensuelles (sinnlich) de la conscience prolétaire (et sur les moyens dont les médias dominants s'approprient et transposent ces besoins de sensualité).

L'insistance sur le corps persiste dans le second volume (et frappe surtout dans le diagnostic à la Bourdieu de la répression d'une culture paysanne du corps par la «distinction» bourgeoise) mais l'analyse de l'imagination obéit à une articulation beaucoup plus complexe. En effet, Negt et Kluge proposent à un moment une sextuple différenciation des coordonnées, ou dimensions, de la conscience sociale: horizontale, verticale, fonctionnelle, irrationnelle, imaginaire et révolutionnaire (p.511). Les deux premières concernent encore les possibilités individuelles immédiates de penser et 
d'organiser une situation donnée, l'observant à travers une large perspective ou, d'un autre côté, la creusant ${ }^{15}$ pour une lecture diachronique ou synchronique; tout comme dans les volumes successifs du Capital, Marx montre que les différents processus peuvent être décrits comme des cycles ou comme des opérations simultanées s'emboîtant les unes dans les autres. Mais les dimensions horizontale et verticale demeurent bornées par l'horizon de la situation immédiate. Ce qui n'est pas entièrement perceptible structurellement dans l'ensemble de coordonnées immédiates -notamment les prolongations multiples des institutions sociales et des entreprises dont l'importance et les activités ne peuvent être déduites sur la base du contact phénoménologique avec leur façade publique - appartient à la catégorie du fonctionnel qui exige différents types de preuves et différents modes de pensée ou d'analyse : "Je dois mesurer pour m'orienter; les formes immédiates de la relation essentielle n'existent plus, sauf si je m'arrange pour les produire moi-même. Par conséquent, l'orientation se trouve dominée par les comportements synthétiques. Les impressions immédiates sont tout aussi trompeuses que l'« évidence » des catégories hégémoniques » (p. 513).

Le fonctionnel est donc déjà le lieu de la réalité aliénée au sens strict du terme ; mais aussi de l'analyse scientifique ou de la rectification de l'apparence et de la déformation idéologique par la théorie. Les trois coordonnées suivantes quant à elles évoluent clairement dans le domaine du «libidinal » ou de l'imagination et de la «subjectivité ». Negt et Kluge construisent ce domaine de manière originale et complexe, élaborant un système triple qui complique utilement et différencie les dualismes habituels (comme l'opposition binaire de Deleuze et Guattari entre le paranoïde et le schizophrénique - le molaire et le moléculaire, le statique et le nomade - et qui est d'une certaine manière trop facilement assimilée à l'opposition de valeurs respectives entre le fasciste et le révolutionnaire). Les connotations philosophiques et psychanalytiques des termes irrationnel et imaginaire, souvent synonymes par ailleurs, disparaissent dès lors qu'ils sont ramenés à leurs sens mathématiques d'origine: irrationnel désigne des quantités qui existent, mais sans aucune incarnation objective dans la réalité (qui ne correspondent à aucun rapport de nombres entiers) et imaginaire des quantités négatives qui doivent être prises en compte dans les calculs mais qui ne peuvent être conçues comme correspondant à des nombres existants : la différence entre les deux pourrait être, par exemple, celle entre $\pi$ et la racine carrée de moins 1 .

Si le fonctionnel est la coordonnée qui me détermine par rapport à l'extérieur, l'irrationnel est la somme des réponses directes, des compromis (ou équilibrages), des dérobades fournis par l'antiréalisme des motifs; l'imaginaire est alors la coordonnée de la perte de réalité, de la perte historique, de la perte d'identité et, ce qui est du plus grand intérêt dans le contexte actuel, de la perte nationale. Mais si la détermination par rapport à l'extérieur [le fonctionnel] comprend encore une dimension de pratique humaine (déformée, mais qui continue de se réaliser), c'est-à-dire du travail aliéné, une vie inversée, une fausse conscience, leur réification et leur élaboration à des niveaux supérieurs, et si l'irrationalité produite par cette dimension et son processus de production reste présente en son sein, la dimension de l'imaginaire est, au contraire, déterminée par le caractère de plus en plus irréel du monde et sa perte d'objectivation. En effet, une attention tournée exclusivement vers le fonctionnel et l'irrationnel, dans le but de protester contre la perte de liens naturels dans les contextes spatiaux et temporels et dans les institutions humaines, a généralement tendance à négliger cette antidimension de la perte de terrain solide et la transformation en néant du temps réel. La 
dimension $d u$ révolutionnaire, aussi vigoureuse simultanément dans toutes les autres dimensions, bien qu'elle ait échoué à s'y accomplir, ne saurait trancher plus par rapport à la dimension particulière de l'anti-monde et de l'imaginaire : l'antagonisme est bien plus prononcé qu'avec le fonctionnel ou l'irrationnel, qui font sans aucun doute barrage au révolutionnaire, mais lui prêtent aussi une nouvelle substance et un nouveau contenu (pp. 511-512).

Ces distinctions seront éclaircies dans la discussion de Negt et Kluge du concept (c'est-àdire de l'expérience) de "réalité » articulé selon l'axe de la détermination (ou de l'indétermination) et de l'objectivité (ou la dissolution des frontières de l'objet) (p. 343). Ainsi que le suggère cependant le passage qui précède, le réel n'a pas un contraire, mais deux : l'anti-réel et l'irréel. Le premier est un refus - par la protestation et la révolte d'accepter la réalité, tandis que le second fait état d'un lien passif et fluctuant avec un monde extérieur fantasmagorique faiblement investi. L'intérêt de ces distinctions est en fin de compte de confronter et d'accepter l'ambivalence de l'engagement politique massif, celui du passé (le mouvement de masse nazi) et celui d'un présent dont tout le monde semble déterminé à nous certifier que les masses actuelles sont devenues de simples consommatrices, ou du moins qu'elles sont définitivement dépolitisées. La remise en question systématique de l'appel lancé avec succès par le nazisme aux classes laborieuses - qui a débuté dans les années 1960 (et est passé souvent par un retour aux premiers travaux de Wilhelm Reich sur le sujet) et constitue la force centrale du motif des ouvrages de Deleuze et Guattari cités plus haut - perd visiblement son sens dès lors qu'on abandonne la distinction entre impulsions réactionnaires et progressistes. Le problème consiste à conserver cette distinction tout en la privant de sa tendance dualistique. De fait, Negt et Kluge conservent l'aspiration vers une transformation utopique de la situation présente par le biais de leur coordonnée " révolutionnaire ", tout en articulant ce qui est généralement appelé « réactionnaire » (sur la base de ses effets) à une myriade de coordonnées différentes qui varie historiquement.

49 En ce qui concerne le présent, les auteurs expliquent à de multiples reprises que Geschichte und Eigensinn est directement issu des réflexions sur le mouvement ouvrier qui ont occupé une grande partie de leur ouvrage précédent (p. 389). Mais même si l'ouvrage ultérieur s'achève lui aussi sur une discussion explicite des différents aspects de la politique de la classe ouvrière, il est clairement conçu pour un niveau théorique plus élevé et une échelle de temps plus longue (pour ne pas dire plus évolutionniste), dépassant la question de la situation actuelle pour aller vers les problèmes de tradition, pédagogie collective, habitus collectif et cicatrices du passé. Ce n'est pas le passage le moins intéressant d'Öffentlichkeit und Erfahrung que celui qui concerne les leçons de la défaite envers laquelle la gauche a bien assez souvent affiché son hypocrisie, quand ce n'était pas son triomphalisme moralisateur. Les deux auteurs nous rappellent ainsi que Mao Zedong qualifiait l'Internationale dans l'un de ses poèmes de "chant tragique », de monument commémorant des défaites sanglants; ils citent aussi le fragment de conclusion de La dialectique de la Raison ${ }^{16}$, tout à fait éloquent: "La bêtise est une cicatrice... La violence subie transforme la bonne volonté en mauvaise volonté », et ils ajoutent :

La sensibilité sociale a néanmoins besoin de la résistance sur laquelle elle travaille et à partir de laquelle elle parvient à se constituer en expérience. Les processus d'apprentissage basés sur l'échec doivent donc être abordés comme deux expériences distinctes: une destructive et une émancipatrice. Lorsque seule l'une des deux est présente, on trouve généralement une erreur d'analyse ${ }^{17}$. 


\section{Apprendre} Lebenslauf (parcours individuel, qu'on retrouve comme titre du premier recueil d'histoires de Kluge), le signal terminologique le plus insistant de toute l'œuvre de Kluge comme de sa collaboration avec Negt. Les trajectoires de vies sont ces unités d'expérience passionnée et existentielle, réduites à des anecdotes glaciales, quasiment statistiques, par le poids même de l'Histoire : une femme fuyant sous les bombes américaines qui se cache dans la cave d'une maison détruite par les bombardements, un professeur de lycée à la retraite qui écrit un mémoire sur Charlemagne pendant la débâcle de mai 1945, des affaires juridiques, des fuites et des vols compulsifs, la survie après guerre et les ruines aussi déroutantes que la richesse inattendue pour les sujets concernés qui semblent souvent, dans les récits et les films de Kluge, confus et dépouillés des habitudes sociales qui auraient donné une certaine stabilité à leurs mouvements, même en l'absence de motivation et d'ambition personnelles. L'anecdote est ici ce qui rattache l'individuel au collectif et à l'historique par la problématisation de ce qui relie: elle ne peut guère donner de leçons, à moins de lever en premier lieu le mystère ultime de la manière dont nous apprenons. Dans ce sens, la seule note optimiste dans le premier long métrage de Kluge (Abschied von Gestern) - «Tu ne peux pas apprendre à ne pas apprendre »- paraitt souvent follement prématurée.

Elle n'en suggère pas moins qu' «apprendre" n'est pas un processus conscient ou volontaire, et que la première étape consiste parfois à découvrir ce qui a été appris, à repérer les forces et les habitudes, les capacités (Arbeitsvermögen) déjà accumulées dans le corps, l'inconscient, la collectivité. La relationalité - qui est, nous l'avons vu, le message pratique ou le slogan ultime de Negt et Kluge - est loin de proposer une synthèse organique de ces capacités; et en effet, leur proposition pour créer des connexions a parfois un ton remarquablement post-moderne :

Mais ces langages multiples des diverses relations ne peuvent être réduits à quoi que ce soit d'unifié. De même que les capacités d'autonomie ne nécessitent pas de traduction complète dans le moindre détail. Elles peuvent parfaitement s'accommoder de l'impossibilité de traduire ces différentes langues les unes dans les autres, et même de leur caractère incompréhensible, à condition que ce qui n'est pas traduit soit appréhendé comme une relation (p. 1088).

Il n'en serait pas moins utile de réfléchir à la relationalité (même ici) à la lumière de la valeur de l'öffentlichkeit affirmée dans le premier texte - le fait de dévoiler au grand jour, d'exprimer et de rendre public, ce qui ne peut être réduit ni à la notion de communication d'Habermas, ni à la simple mise en place d'un nouveau type d'institutions. Le meilleur moyen d'appréhender la liaison entre "relationalité » et « espace public » consiste sans doute à bloquer les deux, ainsi que les limites et frontières édifiées dans notre ordre social actuel :

Pour illustrer la rigidité de la compartimentation sociale de l'expérience, on ne saurait trouver mieux que la prison. Nous y avons, avec l'institution du parloir, l'unique point de contact entre l'expérience de ceux qui sont à l'intérieur et de ceux de l'extérieur, même si en fin de compte les prisonniers appartiennent aux deux. Mais dans l'industrie, ce type de cellule destinée aux visiteurs n'est même pas prévu pour servir de lieu d'échange à ces points de contact dont le travailleur a besoin, en tant qu'organisme dont l'existence indivisible se déroule tout à la fois dedans et dehors (p. 795, n. 5). 

et prendrait aussi la forme d'une politique culturelle au sens large que Negt et Kluge ont déjà donné au terme de révolution culturelle (la culture en tant que telle, ou même la production spécifiquement culturelle, n'est curieusement que très peu discutée dans Geschichte und Eigensinn). discernement (une puissance qui doit être développée indépendamment, comme dans les contes cités en exemples) : elle ne se limite pas à l'évaluation d'une force ou d'une puissance donnée (métier, persévérance, courage, etc.) en soi, mais comprend aussi la manière dont cette puissance interagit avec d'autres forces et les types de combinaisons où elle est appliquée. Ce type de jugement touche au juridique : au-delà du « hasard » de leur profession, le rôle considérable des juges, tribunaux ou jugements dans les récits et les films de Kluge a une signification plus profonde. En effet, une capacité comme la «fiabilité » (Verlässlichkeit) est en fin de compte, dans l'histoire concrète, excessivement ambivalente: associée à d'autres "capacités" contradictoires, le sens du devoir d'un pilote ou d'un mécanicien de bombardier peut figurer dans des récits très différents : d'une part ce qui semble être le traumatisme originel dans les récits de Kluge, le bombardement de sa ville de Halberstadt le 8 avril 1945, alors qu'il avait treize ans ${ }^{18}$, et d'autre part, la «non-fiabilité » du technicien qui, lorsqu'il s'est aperçu que les cibles assignées étaient en fait situées de l'autre côté de la frontière cambodgienne, en a informé Nixon et Kissinger (pp. 700- 703). collective, s'achemine vers l'éthique, mais d'une manière inédite qui se passe désormais du terme et de son histoire pour faire dévier notre attention des qualités putatives des talents personnels et l'orienter vers la question plus urgente de leurs combinaisons. L' Arbeitsvermögen devient ainsi un nouveau moyen de soulever la question, non de la vertu en tant que telle, mais des " vertus » aristotéliciennes originales, dont le livre d'Alisdair Maclntyre After Virtue nous rappelle qu'elles sont multiples et collectives, exigeant de repenser les traditions individualistes de la philosophie morale traditionnelle.

Cette réinvention exigerait de nouveaux processus d'apprentissage auxquels Geschichte und Eigensinn tente de nous proposer des noms nouveaux. C'est un travail qui ne peut cependant être entamé sans une conscience aigüe qu'il existe aussi des Lernprozesse mit tödlichem Ausgang - des processus d'apprentissage à l'issue fatale (titre de l'un des recueils de récits de Kluge). Que d'autres issues, d'autres processus d'apprentissage soient au moins concevables, c'est ce que cet ouvrage nous demande d'imaginer.

\section{NOTES}

1. Öffentlichkeit und Erfahrung: Zur Organisationsanalyse von bürgerlicher und proletarischer Öffentlichkeit (Espace public et expérience), Francfort, Suhrkamp, 1972; Geschichte und Eigensinn, Francfort, Zweitausendeins, 1981. Tous les numéros de page indiqués dans le texte renvoient à ce second ouvrage.

Variations, 21 | 2018 
Ndlr : pour des extraits de ces livres en français, voir O.Negt, L'espace public oppositionnel (textes choisis, introduits et traduits par Alexander Neumann), Payot, 2003.

2. Alexander Kluge et Edgar Reitz, «In Gefahr und grösster Not bringt der Mittelweg den Tod» (Dans le danger et le besoin le plus pressant, la voie médiane est mortelle), Kursbuch, 41 (1975), pp. 42-43.

3. Jean Paulhan, La Preuve par l'étymologie, Paris, Editions de Minuit, 1953.

4. Anton Kaes, Deutschlandbilder, Munich, Text+ Kritik, 1987, p. 48 (disponible aux Harvard University Press sous le titre From Hitler to Heimat).

5. N.d.T.: Cette imperfection se retrouve en français avec la traduction possible du titre par Espace public et expérience. Pour suivre l'usage en vigueur depuis la publication en français en 1986 de l'ouvrage de Jürgen Habermas dont il est question plus loin, L'Espace public, Archéologie de la Publicité comme dimension constitutive de la société bourgeoise, le mot anglais « sphere » employé par Jameson est toujours traduit ici par « espace».

6. Dans les deux livres, Negt et Kluge utilisent le terme prolétarien dans son sens le plus général : "Prolétarien, c'est à dire, séparé des moyens de production, ne désigne pas seulement les caractéristiques du travail du prolétariat industriel, mais toutes les capacités productives qui sont semblablement restreintes » (Geschichte und Eigensinn, p. 445, n. 16).

7. Voir aussi, plus généralement sur Kluge, Miriam Hansen, « Space of History, Language of Time: Kluge's Yesterday Girl (1966) », dans German Film and Literature: Adaptations and Transformations, Eric Rentschler, éd., New York, Methuen, 1985, pp. 193-216 ; « Alexander Kluge, Cinema and the Public Sphere: the Construction Site of Counter-History", Discourse, 6 (1984), pp.53-74 et "The Stubborn Discourse: History and Story-Telling in the Films of Alexander Kluge », dans Persistence of Vision, 2 (automne 1985), pp. 19-29. Voir aussi Andrew Bowie, «Alexander Kluge: An Introduction », Cultural Critique, 4 (automne 1986), pp. 111-118; "Geschichte und Eigensinn », Telos, 66 (hiver 1985-1986), pp. 183-190 et « New Histories: Aspects of the Prose of Alexander Kluge », Journal of European Studies, 12 (1982), pp. 180-208.

8. «Ce n'est qu'en temps de guerre que la brusque liquéfaction du travail historique mort se traduit par une accélération réelle. La cité millénaire de Magdebourg a entièrement brûlé en deux jours pendant la Guerre de Trente ans, mais dès lors qu'il s'agit du processus historique, la Guerre de Trente ans n'a pratiquement provoqué aucun mouvement. En particulier, aucune relation sociale ne s'est véritablement exprimée dans l'incendie de Magdebourg... » (Geschichte und Eigensinn, p. 276).

9. Là encore, leur réflexion trouve un certain écho dans celle de Raymond Williams : l'affirmation d'une utopie urbaine opposée à une utopie pastorale a trouvé ses illustrations les plus riches en science-fiction, notamment dans le «débat » entre Samuel Delany et Ursula Le Guin. Kluge semble cependant avoir modifié sa position anti-urbaine dans Industrialisierung des Bewusstseins, Munich, Piper, 1985.

10. Comme par exemple la description que fait Roland Barthes de l'esthétique artisanale au début de l'époque moderne, notamment chez Flaubert et Baudelaire. Voir Roland Barthes, Le Degré zéro de l'écriture, Paris, Seuil, 1953.

11. Les études contemporaines sur la littérature de l'impérialisme moderne - qui est aussi pour l'essentiel une littérature d'aventure - sont ici très pertinentes. Voir par exemple Edward Said, « Kirn, The Pleasures of lmperialism », Raritan, vol. VII, n² (automne 1987), pp. 27-64.

12. Karl Marx et Friedrich Engels, Manifeste du Parti communiste, Paris, Garnier Flammarion, 1999.

13. Emmanuel Kant, Métaphysique des mœurs, Paris, Flammarion, 1994, p. 152 (cité sous cette forme dans le récit de Kluge "Hinscheiden einer Haltung ", in Lebensläufe, Hambourg, Fischer, 1964, p. 26).

14. Dans Zur Rekonstruktion des historischen Materialismus, Francfort, Suhrkamp, 1976. 
15. Le fait de creuser, qui fait partie de la «capacité de travail» paysanne, est souvent associé dans les contes à la découverte d'un trésor caché : la chance, qui en allemand est exprimée par le même mot que celui qui désigne le bonheur, Glück. Quant au bonheur lui-même, voir les très remarquables pages de Geschichte und Eigensinn, pp. 924- 930, qui prolongent Adorno à bien des égards.

16. "Sur la genèse de la bêtise", in Theodore Adorno et Max Horkheimer, Dialektik der Aufklärung, Francfort, Fischer, 1986, pp. 228-230 (La dialectique de la raison, Paris, Gallimard, 1996, pp. 280-281).

17. Öffentlichkeit und Erfahrung, pp. 404-405.

18. Voir par exemple le « deuxième carnet » de ses Neue Geschichten, Francfort, Suhrkamp, 1977, pp. 33- 106.

INDEX

Mots-clés : Ecole de Francfort, Théorie critique, Oskar Negt, subjectivité rebelle, Eigensinn

\section{AUTEURS}

\section{FREDRIC JAMESON}

Professeur émérite à la Duke University, USA 